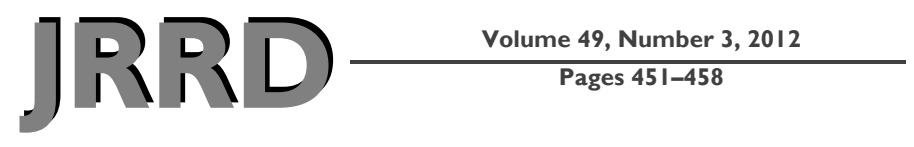

\title{
Single session of brief electrical stimulation immediately following crush injury enhances functional recovery of rat facial nerve
}

\author{
Eileen M. Foecking, PhD; ${ }^{1-2 *}$ Keith N. Fargo, PhD; ${ }^{2-3}$ Lisa M. Coughlin, MD; ${ }^{2}$ James T. Kim, MD; ${ }^{4}$ Sam J. \\ Marzo, MD; ${ }^{1}$ Kathryn J. Jones, PhD $^{1-2,5}$ \\ ${ }^{1}$ Department of Otolaryngology-Head and Neck Surgery, Stritch School of Medicine, Loyola University Chicago, \\ Maywood, IL; ${ }^{2}$ Research \& Development Service, Edward Hines Jr Department of Veterans Affairs Hospital, Hines, \\ $I L ;{ }^{3}$ Department of Molecular Pharmacology and Therapeutics, ${ }^{4}$ Neuroscience Program, and ${ }^{5}$ Department of Microbi- \\ ology and Immunology, Stritch School of Medicine, Loyola University Chicago, Maywood, IL
}

\begin{abstract}
Peripheral nerve injuries lead to a variety of pathological conditions, including paresis or paralysis when the injury involves motor axons. We have been studying ways to enhance the regeneration of peripheral nerves using daily electrical stimulation (ES) following a facial nerve crush injury. In our previous studies, ES was not initiated until $24 \mathrm{~h}$ after injury. The current experiment tested whether ES administered immediately following the crush injury would further decrease the time for complete recovery from facial paralysis. Rats received a unilateral facial nerve crush injury and an electrode was positioned on the nerve proximal to the crush site. Animals received daily 30 min sessions of ES for $1 \mathrm{~d}$ (day of injury only), $2 \mathrm{~d}, 4 \mathrm{~d}, 7 \mathrm{~d}$, or daily until complete functional recovery. Untreated animals received no ES. Animals were observed daily for the return of facial function. Our findings demonstrated that one session of ES was as effective as daily stimulation at enhancing the recovery of most functional parameters. Therefore, the use of a single 30 min session of ES as a possible treatment strategy should be studied in human patients with paralysis as a result of acute nerve injuries.
\end{abstract}

Key words: electrical stimulation, facial nerve, facial paralysis, functional recovery, injury, motoneuron, peripheral nerve, regeneration, repair, therapy.

\section{INTRODUCTION}

Peripheral nerve damage can lead to serious sensory and/or motor dysfunctions. The facial nerve is the most commonly injured cranial nerve. Injury to the facial nerve can have many causes, the most prevalent being Bell palsy, compression or stretching of the nerve caused by tumors or bone fractures, and transection. The facial nerve's susceptibility to injury arises from its complex anatomical course from the brain stem to its target muscles. The severity of facial paresis and the recovery outcome depend on the site of facial nerve injury, with the greater damage observed with an injury more proximal to the cell bodies. Although peripheral nerves are remarkable for their regenerative capacity, recovery from nerve damage can be slow and/or incomplete. Therefore, understanding the factors that can enhance the regenerative process following peripheral nerve damage is important so that therapies can be developed to target such factors.

Applying electrical stimulation (ES) has been shown to affect morphological and functional properties of neurons such as nerve branching, rate and orientation of neurite growth, rapid sprouting, and guidance during axon regeneration [1-4]. Therefore, ES has been explored

\footnotetext{
Abbreviations: ANOVA = analysis of variance, $\mathrm{ES}=$ electrical stimulation.

*Address all correspondence to Eileen M. Foecking, PhD; Edward Hines Jr VA Hospital, Research \& Development Service, 5000 S. 1st Ave, Building 1, Rm A241, Mail 151, Hines, IL 60141; 708-202-5818; fax: 708-202-2327. Email: Eileen.Foecking@va.gov
}

http://dx.doi.org/10.1682/JRRD.2011.03.0033 
as a therapeutic strategy for improving the outcome of peripheral nerve injury. Over the course of several studies, our laboratory has shown that daily ES applied to the facial nerve proximal to the site of injury affects early events in the nerve regeneration process, and therefore functional recovery, in rats by initiating axon sprout formation [5-7]. Pockett and Gavin reported that administering ES following sciatic nerve crush injury in rats accelerates the appearance of the toe-spreading reflex, a sensitive indicator of the onset of motor recovery [8]. Therefore, ES appears to enhance early regeneration events that initiate sprout formation of peripheral nerves in general.

ES is thought to mediate its positive effects on regeneration by enhancing the cell body response. In the presence of low-frequency ES, expression of the neurotrophin brain-derived neurotrophic factor, its high affinity receptor trkB, GAP43, and BII-tubulin significantly increase shortly after femoral nerve injury [9-10]. When administered after femoral nerve transection and surgical repair in rats, as little as $1 \mathrm{~h}$ of ES accelerates axonal growth across the injury site, thereby reducing the time needed for motoneurons to reinnervate muscles [11]. Similar effects of ES have also been demonstrated in the central nervous system. For example, peripheral ES promotes regeneration of the afferent fibers following central injury of dorsal root ganglion axons arising from the sciatic nerve [11].

Our previous studies demonstrating the effectiveness of ES in enhancing recovery from facial nerve crush injury have used daily stimulation beginning the day after injury [5-6]. However, given the evidence that ES acts on early events in the regenerative process, one of the objectives of the current study was to determine the effectiveness of ES administered immediately following crush injury. In addition, because repeated application of direct ES to the facial nerve is not always practical in the clinic, another objective of the study was to determine whether the number of sessions of ES could be reduced while still improving functional recovery.

\section{METHODS}

\section{Animals}

Adult, male, Sprague-Dawley rats ( 2 mo old) were obtained from Harlan (Indianapolis, Indiana) and used for all experiments. Animals were housed under a $12 \mathrm{~h}$ light/dark cycle and received standard rodent diet and water ad libitum.

\section{Surgical Procedures}

For facial nerve crush injuries, all rats received intraperitoneal injections of ketamine $(100 \mathrm{mg} / \mathrm{mL} ; 0.1 \mathrm{~mL} /$ $100 \mathrm{~g}$ body weight) and xylazine $(20 \mathrm{mg} / \mathrm{mL} ; 0.025 \mathrm{~mL} /$ $100 \mathrm{~g}$ body weight). The right facial nerve was exposed and crushed at its exit from the stylomastoid foramen proximal to the bifurcation of the main trunk. The crush injury consisted of two $30 \mathrm{~s}$ crushes using fine forceps, each occurring at the same level of the nerve but with a $180^{\circ}$ change in the orientation of the forceps around the nerve. This method ensured that the entire width of the nerve was symmetrically crushed while leaving the nerve sheath intact to serve as a conduit for regenerating axons. For consistency in amount of pressure applied during the crush, the same researcher crushed all nerves in this study. A successful crush was confirmed in each animal by observing complete loss of the blink reflex, flattening of vibrissae orientation, and paralysis of vibrissae movement. The uninjured left side served as an internal control for each animal.

\section{Electrode Implantation and Electrical Stimulation}

Electrode assemblies were constructed as previously described [5-6]. During the same surgery as the crush procedure, the base of the electrode assembly was sutured onto the paraspinous muscles of each animal. The negative electrode terminal was then sutured approximately $2 \mathrm{~mm}$ proximal to the crush injury site and the positive electrode terminal to connective tissue about 3 to $5 \mathrm{~mm}$ away from the negative terminal on the opposite side of the nerve, such that electrical current passing between the electrode terminals stimulated the regenerating axons in the nerve.

The animals were divided into seven groups ( $n=6$ in all groups): one group that received no ES; one group that received one 30 min session of ES immediately following the injury (1 session); groups receiving one 30 min session of ES immediately following the injury along with additional sessions either $1 \mathrm{~d}$ later (2 sessions), $1 \mathrm{~d}$ to $3 \mathrm{~d}$ later (4 sessions), or $1 \mathrm{~d}$ to $6 \mathrm{~d}$ later (7 sessions); and a group that was stimulated daily until complete recovery as previously described [5-7]. The onset of recovery for both vibrissae orientation and eye blink reflex occurred within a few days. Because of this, the later time points (i.e., more sessions of ES) did not 
always provide an experimental manipulation that was meaningfully different from daily stimulation. In these cases, the later time point groups were incorporated into the daily group.

The animals that received ES were connected to an electrical stimulator (consisting of Stimulus Isolator [Model 850A], Interval Generator [Model 830], Preset Control [Model 832], and Pulse Module [Model 831], World Precision Instruments, Inc; Sarasota, Florida) that generated 1 ms-wide square wave direct current stimulus pulses at $20 \mathrm{~Hz}(1.5 \mathrm{~mA}, 1 \mathrm{~s})$, followed by a $1 \mathrm{~s}$ rest period. This pattern was repeated for $30 \mathrm{~min}$. Voltage was increased slowly and set to a level at which rats displayed a right ear flutter. All animals were placed in the stimulation chamber daily throughout the entire study; however, the ES was only administered to those animals in the 1, 2, 4,7 , or daily session groups for the appropriate number of sessions. The rats in the unstimulated control groups were treated identically in all respects (including being placed in ES chambers for 30 min per day throughout the entire study), except that they received no actual ES.

\section{Behavioral Assessment of Functional Recovery}

To assess recovery following nerve injury, right-side facial functional recovery was compared daily with the uninjured left side. The eye blink reflex, vibrissae movement, and orientation of the vibrissae relative to the rostrocaudal axis of the animal were measured. These parameters were chosen because they are dependent on the facial nerve and have been used in previous studies of facial nerve recovery [5-6,12-13]. The time to complete recovery of all measured parameters was also recorded and analyzed. To ensure the reliability of facial nerve function assessments, the same two observers who were blinded to the treatment groups verified all scores. The facial functional parameters were scored on a 3 -point scale $(1=$ no recovery, 2 = partial recovery, 3 = complete recovery), as previously described [5-6,12-13].

\section{Statistical Analysis}

All outcome measures were analyzed using analysis of variance (ANOVA). In order to test whether more stimulation sessions yielded better outcomes, statistically significant ANOVAs were followed by post hoc analysis of all pairwise comparisons using the Holm-Sidak method to correct for multiple comparisons.

\section{RESULTS}

\section{Eye Blink Reflex}

Time to onset of recovery of the eye blink reflex was significantly reduced by $\operatorname{ES}(F(3,30)=7.13, p<0.001$; Figure 1(a)). Mean time to onset in untreated animals was $7.0 \mathrm{~d}$, but this was significantly reduced in all electrically stimulated groups $(p<0.05)$. Among stimulated animals, mean time to onset of eye blink recovery was $4.2 \mathrm{~d}$ ( $\sim 40 \%$ reduction). This time did not differ significantly between electrically stimulated groups, with a single session of ES being just as effective as multiple sessions. Since onset of the eye blink reflex was recovered in the 4 and $7 \mathrm{~d}$ ES treatment groups before $4 \mathrm{~d}$, these animals were included in the daily stimulation group.

Untreated animals completely recovered the eye blink reflex in a mean of $17.0 \mathrm{~d}$. Complete eye blink recovery time was also significantly affected by ES ( $F(5$, $31)=2.59, p<0.05$; Figure 1(b)). Animals receiving 1, 2, or 4 sessions of ES completely recovered the eye blink reflex in a mean of $12.5 \mathrm{~d}$ ( $\sim 26 \%$ reduction); these groups were all significantly reduced compared to untreated animals ( $p<0.05)$, but did not differ from one another. Animals receiving 7 sessions or daily stimulation recovered full eye blink reflex in an intermediate $14.6 \mathrm{~d}$; these groups did not differ significantly from either untreated animals or the other stimulation groups.

\section{Vibrissae Orientation}

The mean time to recovery of vibrissae orientation in untreated animals was $12.6 \mathrm{~d}$, and this was significantly reduced by $\operatorname{ES}(F(4,32)=20.53, p<0.05$; Figure 2$)$. All groups receiving ES recovered normal vibrissae orientation more quickly than animals that did not receive ES ( $p<$ 0.05 ). Animals in the 1,2 , and 4 session groups recovered in $9.1 \mathrm{~d}$ ( $\sim 28 \%$ reduction), on average, and these groups did not differ significantly from one another. However, animals in the daily stimulation group recovered more quickly than animals receiving fewer sessions of stimulation $(p<0.05)$, with a mean recovery time of only $5.5 \mathrm{~d}$ ( $\sim 56 \%$ reduction from untreated controls).

\section{Vibrissae Movement}

The mean time to the onset of vibrissae movement in untreated animals was $17.1 \mathrm{~d}$. This time was significantly 

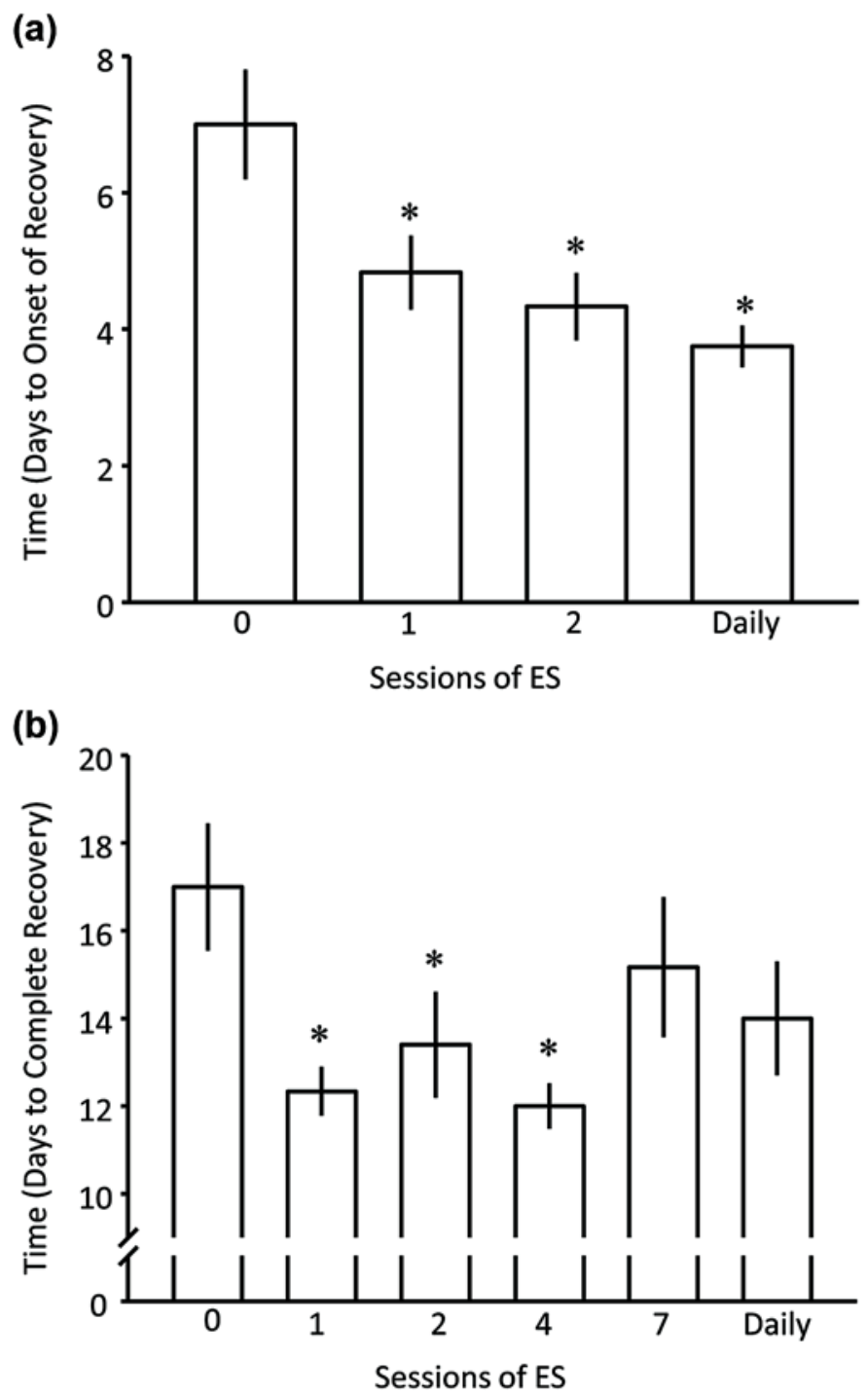

Figure 1.

Number of days to (a) onset of recovery or (b) complete recovery of eye blink reflex for untreated animals, animals receiving either $1,2,4$, or 7 sessions of electrical stimulation (ES), and animals receiving daily stimulation until complete recovery. ES administered for any number of sessions significantly reduced time to onset of recovery of eye blink. Time to complete recovery of eye blink reflex was significantly reduced in animals that received 1 , 2 , or 4 sessions of ES compared with untreated animals, whereas 7-session and daily treatment groups recovered in intermediate period of time that was not significantly different either from other stimulation groups or from untreated animals. Bar heights represent mean \pm standard error of mean. ${ }^{*}$ Significantly different from untreated controls, $p<0.05$.

reduced by $\operatorname{ES}(F(5,34)=3.56, p<0.05$; Figure 3(a)), with all groups that received ES having a lower time to onset than untreated animals $(p<0.05)$. Among animals

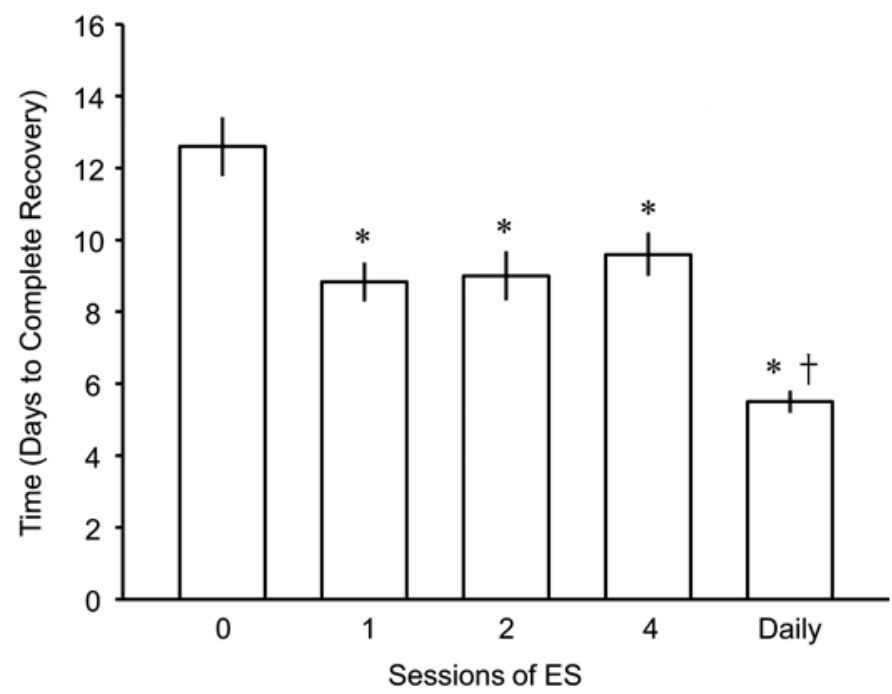

Figure 2.

Number of days to return of normal vibrissae orientation for untreated animals, animals receiving either 1, 2, or 4 sessions of electrical stimulation (ES), and animals receiving daily stimulation until complete recovery. Time to return of normal vibrissae orientation was significantly reduced in all ES groups. Bar heights represent mean \pm standard error of mean. ${ }^{*}$ Significantly different from untreated controls, $p<0.05$. 'Significantly different from all other groups, $p<0.05$.

receiving ES, mean time to onset was $13.2 \mathrm{~d}$ ( $23 \%$ reduction), and this did not differ significantly between groups, with a single session of stimulation being just as effective as multiple sessions.

Time to full recovery of vibrissae movement showed a similar pattern. Untreated animals recovered full vibrissae movement in a mean of 20.4 d, with ES significantly reducing this time $(F(5,32)=4.62, p<0.01$; Figure $3(\mathbf{b}))$. All groups receiving ES recovered full vibrissae movement more quickly than untreated animals $(p<0.05)$. Among electrically stimulated animals, mean time to recovery was $16.0 \mathrm{~d}$ ( $\sim 22 \%$ reduction). This did not differ between electrically stimulated groups, with a single session being just as effective as multiple sessions.

\section{Complete Recovery}

The time to complete overall recovery was also measured and scored as the day on which each individual animal had reached complete recovery of all other behavioral parameters. Mean time to complete recovery in untreated animals was $20.4 \mathrm{~d}$. This time was significantly affected by $\operatorname{ES}(F(5,30)=3.79, p<0.01$; Figure 4). With the exception of the 7-session group, mean time to complete recovery in 
(a)

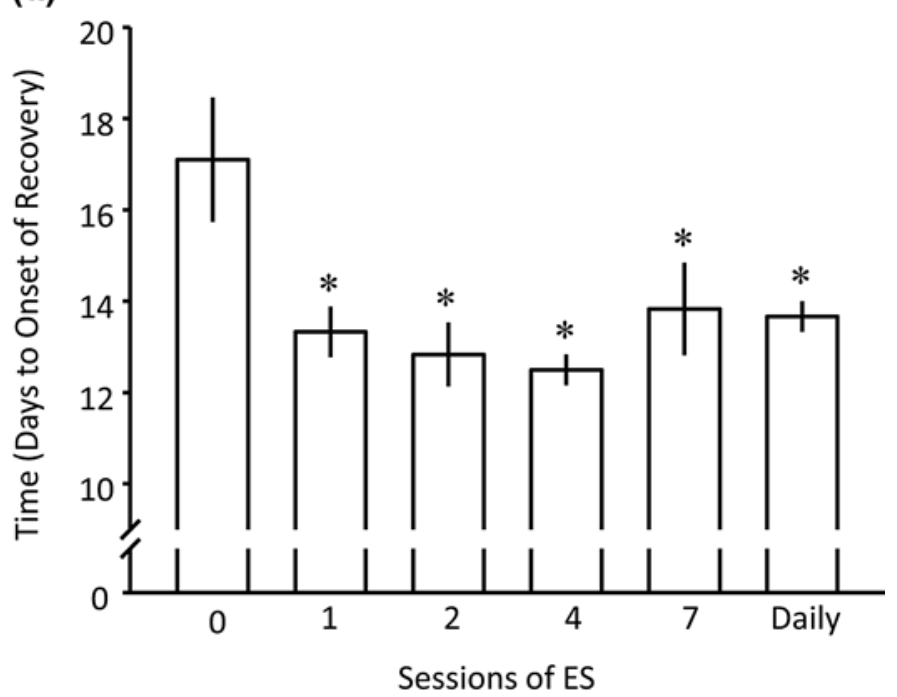

(b)

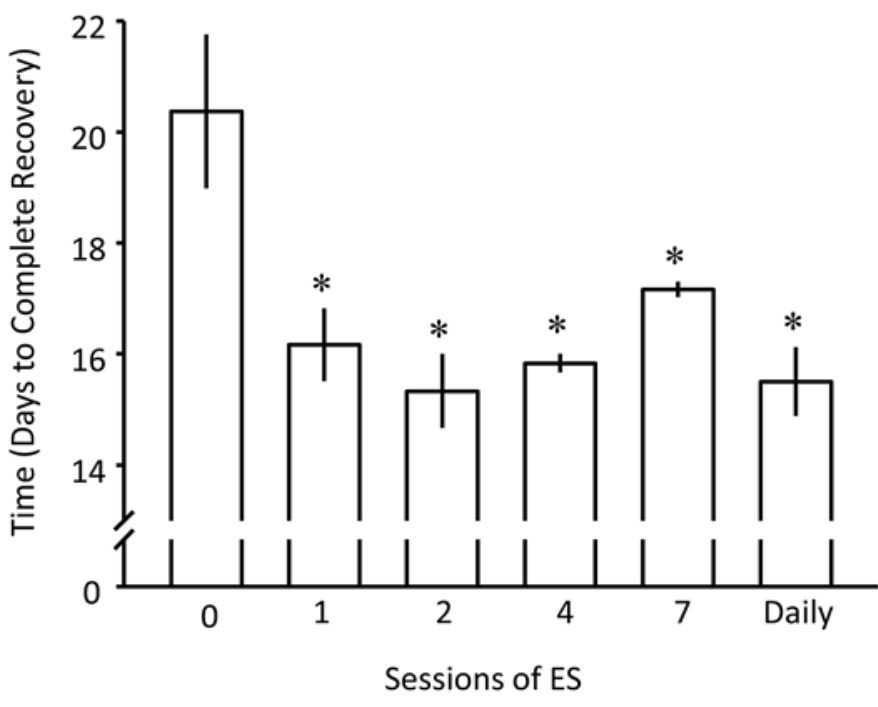

Figure 3.

Number of days to (a) onset or (b) complete recovery of vibrissae movement for untreated animals, animals receiving either $1,2,4$, or 7 sessions of electrical stimulation (ES), and animals receiving daily stimulation until complete recovery. Time to onset and time to complete recovery were both significantly reduced in all ES groups, and single session of ES was just as effective as multiple sessions. Bar heights represent mean \pm standard error of mean. ${ }^{*}$ Significantly different from untreated controls, $p<0.05$.

animals receiving stimulation was $15.8 \mathrm{~d}(\sim 23 \%$ reduction, $p<0.05$ ), and this did not differ between groups, with a single session being just as effective as multiple sessions. Animals receiving 7 sessions of ES reached complete

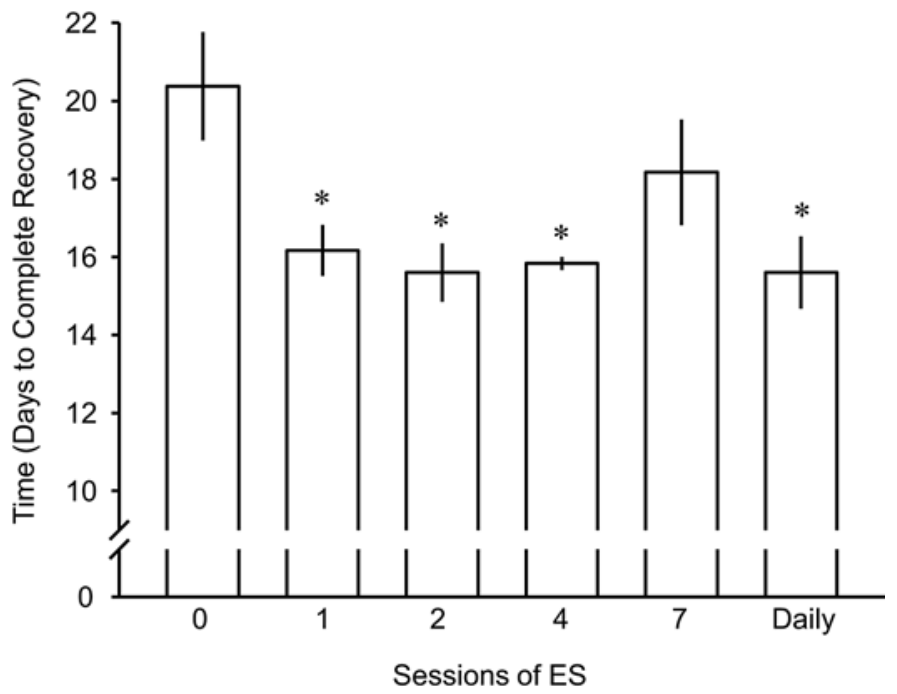

Figure 4.

Number of days to complete recovery of all measured functional parameters for untreated animals, animals receiving either 1, 2, 4 , or 7 sessions of electrical stimulation (ES), and animals receiving daily stimulation until complete recovery. Time to complete recovery was significantly reduced by ES for animals in the 1, 2, and 4 session groups and the daily stimulation group, and single session of ES was just as effective as multiple sessions. For animals in the 7-session group, complete recovery occurred at an intermediate time point that was not significantly different from either untreated animals or animals in other stimulation groups. Bar heights represent mean \pm standard error of mean. ${ }^{*}$ Significantly different from untreated controls, $p<0.05$.

recovery in a mean of $18.2 \mathrm{~d}$, an intermediate number that did not differ from either untreated animals or the other stimulated groups.

\section{DISCUSSION}

Recently, our laboratory has focused on developing therapies that enhance axonal regeneration following nerve injury. Our initial studies investigated the therapeutic potential of ES in nerve regeneration following a rat facial nerve crush injury because of ES's enhancing effects on the morphological and functional properties of neurons [1-4]. In these studies, daily, low-frequency ES hastened the onset of recovery of the eye blink reflex by reducing the initial delay in sprout formation rather than by increasing the rate of axonal regeneration, indicating that ES acts primarily on events in the early stages of recovery [5-7]. Although early recovery parameters were enhanced with the administration of ES starting the day 
following injury, complete recovery of all functional parameters was not significantly affected. Therefore, the present study set out to determine whether ES, if given immediately following a facial nerve injury, can significantly decrease time to complete functional recovery. Additionally, since brief ES applied to an injured femoral nerve has been shown to accelerate axonal growth across the injury site [11], an additional goal of the current study was to determine whether daily stimulation is necessary or whether a smaller number of sessions would be sufficient to enhance regeneration and recovery in the facial nerve injury model.

ES of nerve terminals has been shown to induce rapid filopodial sprouting in primary neuron cell cultures in the presence of extracellular $\mathrm{Ca}^{2+}$ [3]. It has also been reported that, following sciatic nerve crush injury in rats, administering ES accelerates the appearance of the toespreading reflex, a sensitive indicator of the onset of motor recovery [8], further supporting the concept that ES enhances early regeneration events that initiate sprout formation. Therefore, the current study first tested whether ES administered immediately following injury rather than the following day would be sufficient to significantly enhance complete functional recovery. Building on our previous findings (in which ES was administered beginning on the day following injury), this report demonstrates that immediate ES administration, regardless of the number of sessions, significantly reduces the time to complete recovery of all functional parameters. The average time to complete recovery was approximately $20 \mathrm{~d}$ for untreated animals and approximately $16 \mathrm{~d}$ for animals treated with ES, showing a 20 percent reduction in recovery time. These data further support a role for ES in mediating early events in the regenerative process.

Our previous work with ES used a daily exposure paradigm. In a femoral nerve injury model, however, brief ES-as little as $1 \mathrm{~h}$-has been shown to accelerate axonal growth across the injury site, thereby reducing the time needed for motoneurons to reinnervate muscles [11]. Therefore, the present experiment also studied whether daily ES was necessary or whether a shorter number of sessions would be sufficient to enhance regeneration following a facial nerve crush injury. When compared with animals that did not receive ES, a single 30 min session of ES was as effective as more prolonged stimulation regimens in reducing time to functional recovery of the onset of the eye blink and both the onset and complete recovery of the vibrissae movement. In fact, increasing the number of sessions or even administering ES daily appears to be detrimental to recovery time for the complete eye blink. In the most critical measurement, complete recovery, a single bout of ES was no better or worse than daily stimulation.

One potential mechanism for the actions of ES may be alterations of the inflammatory process. ES may reduce inflammation sooner or may inhibit the inflammatory process from proceeding to a point of secondary damage after the crush injury. Although ES is known to alter the mobility of macrophages and Schwann cells at the site of injury, we do not believe this affects functional recovery. We have previously explored the role of inflammation on functional recovery by treating with the corticosteroid prednisone following an intratemporal facial nerve crush injury [14]. We determined that prednisone does not affect the recovery time either alone or in combination with ES, suggesting that the effects of ES on functional recovery are not through an inflammatory mechanism. Additionally, others have looked at a short-term, high-dose methylprednisolone treatment following optic nerve injury in rats and found that such treatment has no effect on cell survival, macrophage activity at the site of injury, axonal regeneration, or visual function [15].

\section{CONCLUSIONS}

These findings are the first to show that a single brief (30 min) session of ES, when given immediately after the facial nerve crush injury, can significantly reduce time to complete functional recovery. Gordon et al. have shown that brief ( $1 \mathrm{~h}$ ) low-frequency ES given to patients within $15 \mathrm{~min}$ of an open carpal tunnel release surgery increased the number of motor units, resulting in increased muscle reinnervation as early as 3 mo postsurgery. Muscle reinnervation was significantly higher after 6 to 8 and 12 mo postsurgery [16]. These findings have exciting clinical implications for facial nerve injuries because they suggest that, if a surgeon identifies a facial nerve injury during a surgical procedure, a single brief session of ES administered to the nerve prior to wound closure may increase muscle reinnervation and therefore decrease the patient's recovery time. A single session of direct nerve stimulation may not always be feasible, as in cases of facial paralysis resulting from Bell palsy. Therefore, we plan to further elucidate the molecular mechanism underlying the effects of ES on functional recovery and 
explore potential pharmacological agents that may mimic the actions of ES for use in situations where direct nerve stimulation is not possible.

\section{ACKNOWLEDGMENTS}

\section{Author Contributions:}

Study concept and design: E. M. Foecking. Acquisition of data: E. M. Foecking, L. M. Coughlin, J. T. Kim. Analysis and interpretation of data: E. M. Foecking, K. N. Fargo. Drafting of manuscript: E. M. Foecking, K. N. Fargo. Critical revision of manuscript for important intellectual content: S. J. Marzo, K. J. Jones.

Statistical analysis: K. N. Fargo.

Obtained funding: E. M. Foecking, K. J. Jones.

Study supervision: S. J. Marzo, K. J. Jones.

Financial Disclosures: The authors have declared that no competing interests exist.

Funding/Support: This material was based on work supported by the Research Funding Committee at Loyola University (grant LU\# 200374) and the Department of Veterans Affairs Rehabilitation Research and Development Service (grant CDA-2 RR\&D B6598W).

Additional Contributions: Dr. Jones is now with the Department of Anatomy and Cell Biology, Indiana University School of Medicine and Richard L. Roudebush Department of Veterans Affairs Medical Center, Indianapolis, Indiana. Dr. Coughlin is now with the Department of Surgery, University of Toledo, Toledo, Ohio. Dr. Kim is now with the Department of Internal Medicine, University of Chicago Hospitals, Chicago, Illinois.

Institutional Review: All surgical procedures were performed in accordance with the National Institutes of Health guidelines and approved by the Institutional Animal Care and Use Committee.

\section{REFERENCES}

1. Borgens RB. Electrically mediated regeneration and guidance of adult mammalian spinal axons into polymeric channels. Neuroscience. 1999;91(1):251-64. [PMID:10336075] http://dx.doi.org/10.1016/S0306-4522(98)00584-3

2. Borgens RB, Roederer E, Cohen MJ. Enhanced spinal cord regeneration in lamprey by applied electric fields. Science. 1981;213(4508):611-17. [PMID:7256258] http://dx.doi.org/10.1126/science.7256258

3. Manivannan S, Terakawa S. Rapid sprouting of filopodia in nerve terminals of chromaffin cells, PC12 cells, and dorsal root neurons induced by electrical stimulation. J Neurosci. 1994;14(10):5917-28. [PMID:7931553]

4. Patel N, Poo MM. Orientation of neurite growth by extracellular electric fields. J Neurosci. 1982;2(4):483-96.

[PMID:6279799]
5. Lal D, Hetzler LT, Sharma N, Wurster RD, Marzo SJ, Jones KJ, Foecking EM. Electrical stimulation facilitates rat facial nerve recovery from a crush injury. Otolaryngol Head Neck Surg. 2008;139(1):68-73. [PMID:18585564] http://dx.doi.org/10.1016/j.otohns.2008.04.030

6. Hetzler LE, Sharma N, Tanzer L, Wurster RD, Leonetti J, Marzo SJ, Jones KJ, Foecking EM. Accelerating functional recovery after rat facial nerve injury: Effects of gonadal steroids and electrical stimulation. Otolaryngol Head Neck Surg. 2008;139(1):62-67. [PMID:18585563] http://dx.doi.org/10.1016/j.otohns.2008.02.006

7. Sharma N, Coughlin L, Porter RG, Tanzer L, Wurster RD, Marzo SJ, Jones KJ, Foecking EM. Effects of electrical stimulation and gonadal steroids on rat facial nerve regenerative properties. Restor Neurol Neurosci. 2009;27(6): 633-44. [PMID:20042787]

8. Pockett S, Gavin RM. Acceleration of peripheral nerve regeneration after crush injury in rat. Neurosci Lett. 1985; 59(2):221-24. [PMID:3877256] http://dx.doi.org/10.1016/0304-3940(85)90203-4

9. Al-Majed AA, Brushart TM, Gordon T. Electrical stimulation accelerates and increases expression of BDNF and trkB mRNA in regenerating rat femoral motoneurons. Eur J Neurosci. 2000;12(12):4381-90. [PMID:11122348]

10. Al-Majed AA, Tam SL, Gordon T. Electrical stimulation accelerates and enhances expression of regenerationassociated genes in regenerating rat femoral motoneurons. Cell Mol Neurobiol. 2004;24(3):379-402. [PMID:15206821] http://dx.doi.org/10.1023/B:CEMN.0000022770.66463.f7

11. Brushart TM, Hoffman PN, Royall RM, Murinson BB, Witzel C, Gordon T. Electrical stimulation promotes motoneuron regeneration without increasing its speed or conditioning the neuron. J Neurosci. 2002;22(15):6631-38. [PMID:12151542]

12. Kujawa KA, Kinderman NB, Jones KJ. Testosteroneinduced acceleration of recovery from facial paralysis following crush axotomy of the facial nerve in male hamsters. Exp Neurol. 1989;105(1):80-85. [PMID:2744130] http://dx.doi.org/10.1016/0014-4886(89)90174-X

13. Tanzer L, Jones KJ. Neurotherapeutic action of testosterone on hamster facial nerve regeneration: Temporal window of effects. Horm Behav. 2004;45(5):339-44. [PMID:15109908] http://dx.doi.org/10.1016/j.yhbeh.2004.01.002

14. Sharma N, Moeller CW, Marzo SJ, Jones KJ, Foecking EM. Combinatorial treatments enhance recovery following facial nerve crush. Laryngoscope. 2010;120(8):1523-30. [PMID:20641084] http://dx.doi.org/10.1002/lary.20997

15. Ohlsson M, Westerlund U, Langmoen IA, Svensson M. Methylprednisolone treatment does not influence axonal regeneration or degeneration following optic nerve injury 
JRRD, Volume 49, Number 3, 2012

in the adult rat. J Neuroophthalmol. 2004;24(1):11-18. [PMID:15206432]

http://dx.doi.org/10.1097/00041327-200403000-00003

16. Gordon T, Brushart TM, Chan KM. Augmenting nerve regeneration with electrical stimulation. Neurol Res. 2008; 30(10):1012-22. [PMID:19079975]

http://dx.doi.org/10.1179/174313208X362488

Submitted for publication March 2, 2011. Accepted in revised form August 12, 2011.
This article and any supplementary material should be cited as follows:

Foecking EM, Fargo KN, Coughlin LM, Kim JT, Marzo SJ, Jones KJ. Single session of brief electrical stimulation immediately following crush injury enhances functional recovery of rat facial nerve. J Rehabil Res Dev. 2012; 49(3):451-58.

http://dx.doi.org/10.1682/JRRD.2011.03.0033

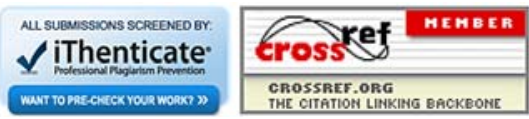

